Published in final edited form as:

J Am Geriatr Soc. 2016 September ; 64(9): 1858-1862. doi:10.1111/jgs.14233.

\title{
A Comparison of Frailty Phenotypes for Prediction of Mortality, Incident Falls, and Hip Fractures in Older Women
}

\author{
Oleg Zaslavsky, PhD ${ }^{1}$, Shira Zelber-Sagi, PhD², Shelly L. Gray, PharmD ${ }^{3}$, Andrea Z. \\ LaCroix, $\mathrm{PhD}^{4}$, Robert L. Brunner, $\mathrm{PhD}^{5}$, Robert B. Wallace, $\mathbf{M D}^{6}$, Mary J. O'Sullivan, MD ${ }^{7}$, \\ Barbara Cochrane, $\mathbf{P h D}^{1}$, and Nancy F. Woods, PhD $^{1}$ \\ ${ }^{1}$ School of Nursing, University of Washington, Seattle, WA \\ ${ }^{2}$ Faculty of Health Sciences and Social Welfare, University of Haifa, Israel \\ ${ }^{3}$ School of Pharmacy, University of Washington, Seattle, WA \\ ${ }^{4}$ Division of Epidemiology, School of Medicine, University of California San Diego, CA \\ ${ }^{5}$ Department of Nutrition, University of Nevada, Reno, NV \\ ${ }^{6}$ College of Public Health, University of lowa, lowa City, IA \\ ${ }^{7}$ Miller School of Medicine, University of Miami, Miami, FL
}

\section{Abstract}

Objectives-We compared the ability of the commonly used Women's Health Initiative (WHI) and Cardiovascular Health Study (CHS) frailty phenotypes to predict falls, hip fracture, and death in WHI Clinical Trial participants aged $\searrow 65$.

Design-Longitudinal cohort study.

Setting-Women's Health Initiative Clinical Trial (WHI CT)

Participants-3,558 participants with data on both WHI and CHS frailty phenotypes

Methods-CHS frailty criteria included weight loss, poor energy, weakness, slowness, and low physical activity. WHI operationalized frailty similarly, but with the RAND-36 physical function scale substituted for slowness and weakness. Thresholds of $<13$ (2-point), 13-78 (1-point) and $>78$ ( 0 points) on the physical function scale were used to indicate levels of functioning. A summary score of $\geq 3$ across frailty criteria was used to define frailty, 2 or 1 was used to define prefrailty, and a score of 0 indicated nonfrailty. Outcomes were modelled using Cox regressions. Harrell's Cstatistics were compared for models containing alternative instruments.

Address correspondence to Oleg Zaslavsky, PhD, Department of Biobehavioral Nursing and Health Systems, School of Nursing, University of Washington, Seattle, WA, oleg.zaslavsky @ fulbrightmail.org, Phone number: (206) 849-3301.

Conflict of Interest: The editor in chief has reviewed the conflict of interest checklist provided by the authors and has determined that the authors have no financial or any other kind of personal conflicts with this paper.

Author Contributions: Drs. Zaslavsky and Zelber Sagi had full access to all of the data used in the study and take responsibility for the integrity of the data and accuracy of data analysis. Zaslavsky, Woods, Zelber-Sagi: study concept, design, interpretation of data, and preparation of manuscript. Zaslavsky, Zelber-Sagi, Woods: data analysis and visualization. Gray, LaCroix, Brunner, Wallace, O'Sullivan, Cochrane: preparation of manuscript. 
Results-About 5\% were frail based on either CHS or WHI phenotypes. WHI frailty phenotype was associated with higher rates of falls (Hazard Ratio $[\mathrm{HR}]=1.48, p=0.003$ ), hip fracture (HR=1.87, $p=0.04)$, and death (HR=2.32, $p<0.001)$. Comparable HRs in CHS-phenotype frail women were $1.32(p=0.04), 1.08(p=0.83)$, and $1.91(p<0.001)$, respectively. Harrell's C-statistics revealed marked but insignificant differences in predicting abilities between CHS and WHI phenotype models ( $p>0.5$ for all)

Conclusion-The WHI phenotype, which does not require direct measurements of physical performance, might offer a practical advantage for epidemiological and clinical needs.

\section{Keywords}

frailty; SF-36; function; mortality; falls; hip fracture; predictive ability

\section{Introduction}

Based on data from the Cardiovascular Health Study (CHS), a phenotype of frailty was proposed in which three or more of five indicators are present: slowness, weakness, fatigue, low physical activity, and body-weight loss (1). Although the predictive validity of the CHS phenotype has been repeatedly confirmed (1-4), a refinement not requiring direct measurement of physical performance would have practical advantages in large epidemiological studies, in populations with chronic disease $(5,6)$ and in continuous-care settings (4). Consequently, the Women's Health Initiative (WHI) offered a frailty phenotype in which a score lower than 75 on the RAND-36 physical function scale replaced the CHS criteria of slowness and weakness (4). An overall agreement between the CHS and WHI screening instruments for frailty was fair (5) and hence additional steps were undertaken to harmonize between two measuring schemes. Johansen et al., 2014 identified thresholds for self-reported function that were more closely aligned with performance-based scores. Although the modification yielded a substantial improvement in agreement between two instruments (5), there has not yet been a direct comparison of the WHI and CHS criteria in the context of their predictive ability of common geriatric outcomes such as falls, hip fracture, and death. We aimed to compare the modified WHI phenotype with the CHS phenotype for prediction of falls, hip fracture, and death in a subsample of the WHI Clinical Trial (CT) participants aged 65 years or older for whom data on both phenotypes were available. We hypothesized that the CHS and WHI phenotypes would have similar predictive abilities.

\section{Method}

\section{Sample}

The WHI CT $(N=68,132)$ is a study of postmenopausal women's health. It was designed as a set of randomized CTs. Details of the design, data collection methods, and baseline data are available elsewhere (7). Briefly, women aged 50 to 79 years were recruited from 1993 to 1998 at 40 clinical centers in the United States. Women were excluded for predicted survival of 3 years or less; for conditions limiting adherence or for active participation in other trials. A random $25 \%$ subsample $(N=6,025)$ of participants aged 65 years and older attended 
baseline and Year 1 clinical visits in which self-report and physical performance measures were collected. From this subsample, 3,666 (60.9\%) women provided complete data on CHS frailty components (defined later), 3,865 (64.2\%) individuals had non-missing data on WHI frailty criteria (defined later) and 3,558 (59\%) participants had provided complete data on both CHS and WHI frailty elements, composing the analytical sample used here. Detailed description of data availability per CHS and WHI frailty phenotypes is provided in supplementary materials (see Figure S1). All WHI participants provided written informed consent, and institutional review board approval was obtained at each of the $40 \mathrm{WHI}$ clinical centers and at the Clinical Coordinating Center.

\section{Measurements}

Physical function was measured using the RAND-36 (8) physical function scale. The scale includes 10 self-report items measuring limitation in the activities of daily living. The scale uses 0-100 scoring, with higher scores indicating better functioning. The WHI Physical Activity scale included a self-report of frequency and duration of mild, moderate, and strenuous activities, with total expenditure of energy calculated as a metabolic equivalent task score (METs). Two other RAND-36 items (from the Vitality scale) inquire about a participant's feeling of being worn out or tired in the previous 4 weeks; these items are intended to indicate exhaustion. At the baseline and Year 1 follow-up visit, trained staff clinically collected anthropometric measurements such as weight and height. These were used to compute BMI. Tests of physical performance included grip strength (using a handheld Jamar dynamometer, expressed in $\mathrm{kg}$ ) and walking speed (time in seconds to walk $6 \mathrm{~m}$ at usual pace, expressed as $\mathrm{m} / \mathrm{s}$ ). Two trials were conducted for performance based measures and scored as the average of the two trials.

\section{CHS Phenotype}

Frailty was operationally defined, using the CHS definition (1), as the presence of three or more of the following criteria:

1. Shrinking, indicated by either objectively measured weight loss of 10 pounds or more or weight loss of $5 \%$ or more between the baseline and Year 1 visit.

2. Weakness, indicated by grip strength in the lowest quintile stratified according to BMI categories (see Supplementary Table 1).

3. Exhaustion, indicated by RAND-36 self-report of being tired or worn out at least most of the time (see Supplementary Table 1).

4. Slowness, indicated by a gait speed less than or equal to $0.8 \mathrm{~m} / \mathrm{s}$. This threshold was found to be a sensitive marker of mobility impairment $(9,10)$.

5. Low physical activity, indicated by a WHI Physical Activity questionnaire score in the lowest quintile.

For each criterion, one point was assigned if the participant's assessment was below the criterion-specific cut-point. This yielded a score ranging from 0 to 5 . Consistent with other 
studies, a score of 3 or more was used to define frailty; 2 or 1 was used to define prefrailty, and a score of 0 indicated nonfrailty.

WHI Frailty Phenotype-The WHI operationalization of frailty included the same criteria for shrinking (weight loss of $\geq 10$ pounds or $\geq 5 \%$ between the baseline and Year 1 visit), exhaustion (RAND-36 self-report of being tired or worn out at least most of the time) and physical activity (WHI Physical Activity questionnaire score in the lowest quintile). The main difference is that the RAND-36 physical function scale (10 items) substituted for slowness and weakness criteria. A threshold of 13 or less on the RAND-36 physical function scale was used to indicate poor physical function ( 2 points); a score of 13-78 indicated intermediate function (1 point) and a score of more than 78 was indicative of a good physical function (0 points; [5]). Similar to the CHS definition, 0 to 5 summary scores were calculated. Women having three or more of the five points were considered to be frail, those with one or two were considered to be prefrail, and those with none were considered to be nonfrail.

\section{Ascertainment and Adjudication of Death, Hip Fractures, and Falls}

Participant deaths (and other outcomes) were ascertained through annual mailed medical updates and periodic checks of the National Death Index (NDI) for all participants. At the time of this analysis, the latest WHI mortality data were available through December 1, 2013. Hip fractures and falls were also ascertained from the annual medical updates, with hip fractures adjudicated by WHI physicians using medical records. Participants reported the number of times they fell or landed on the ground in the interval since the completion of the last medical-update form. We included 2 years of follow-up for falls after the Year 1 clinical visit in which a participant was identified as having an incident fall if she had reported at least one fall per year. Because our goal was to examine incidence of hip fracture and falls subsequent to ascertainment of frailty, we excluded hip fractures and falls that were reported before the first annual follow-up visit. Information on fractures was included for the duration of the study follow-up.

\section{Statistical Analysis}

Year 1 statistics for age and individual frailty criteria were examined using CHS and WHI frailty phenotypes. Frequencies were estimated for categorical variables, and means and standard deviations $(S D S)$ were calculated for continuous variables. Cox proportional hazard models were used to estimate the association of frailty and prefrailty criteria according to CHS or WHI phenotypes on all-cause mortality, hip fractures, or falls using the nonfrailty category as a reference group. Models were adjusted for age. The time-to-event was defined as the number of years (in resolution of days) from WHI Year 1 follow-up visit to study outcomes.

To compare the predictive ability of the CHS and WHI frailty phenotypes, we calculated Harrell's C indexes (11), which estimated the probability of concordance between observed and predicted responses for the mortality, hip-fracture, and falls models, including each phenotype separately. For the purpose of estimating reliable confidence intervals (CIs), we divided the main analytical sample randomly into derivation and validation sets of equal 
size. Age-adjusted models were run in the derivation set, and C-indexes and 95\% CIs were then estimated in the validation set (12). Finally, C-indexes test statistics were compared by estimating their differences, generating a 95\% CI of the differences, and then specifying whether that $\mathrm{CI}$ included zero. All statistical analysis was completed using STATA, version 11.2 (StataCorp, College Station, TX)

\section{Results}

\section{Characteristics of the Study Population and Concordance Between the CHS and WHI Phenotypes}

Characteristics of the cohort of 3,558 older women (average age 70.9 years) are shown in Table 1. About $5 \%$ were frail based on either CHS or WHI phenotypes. Comparing individual criteria, slowness and weakness was identified in $11 \%$ and $27 \%$ of study participants, respectively, whereas total low and intermediate physical function was identified in $42 \%$ of the sample.

Classification of frailty status using the phenotypes was concordant in 2,597 (73\%) women. The kappa statistic was $0.5(p<0.001)$. The Spearman correlation between the CHS and WHI phenotypes was $0.58(p<0.001)$.

\section{WHI Phenotype Versus the CHS Phenotype for Prediction of Risk of Falls, Hip Fracture, and Mortality}

During an average follow-up of 12 years $(S D=3.9)$ after the Year 1 clinical visit, $749(21 \%)$ older women died and 157 (4\%) experienced hip fracture. A more proximal follow-up of an average of 2.6 years yielded an overall incident fall rate of $27.6 \%$.

All-cause mortality rates were higher with greater levels of frailty using either the CHS or WHI phenotypes (Table 3). Point estimates for hazard ratios (HRs) were higher for the WHI phenotype model. Specifically, women in the prefrail group had a 1.6 times higher death rate $(p<0.001)$ than nonfrail women, and those with frailty had a 2.3 higher death rate $(p<$ 0.001 ). Models had marked, but insignificant differences in predictive ability in terms of $\mathrm{C}$ index. The estimates were 0.61 and 0.63 for CHS and WHI phenotypes respectively ( $p$ for comparison $=0.06$ ).

Frailty identified using the CHS and WHI phenotypes revealed different rates of hip fracture. After adjustment for age, women with frailty identified by the CHS phenotype had a 1.1 higher rate of hip fracture, not significant ( $p=0.83$ ); a comparable HR in the WHI phenotype model indicated a 1.9 higher hazard of fracture $(p=0.04)$. There was no significant difference in the $\mathrm{C}$ index between the two models $(p$ for comparison $=0.14$ )

Finally, the relationship between higher levels of frailty and rates of fall was also different in the CHS and WHI phenotypes, so that women with prefrailty by WHI phenotype had 1.2 times higher hazards of falls ( $p<0.003$ ); prefrailty by CHS phenotype yielded insignificant estimates ( $\mathrm{p}=0.62)$. Women in the frail group based on the WHI phenotype had a 1.5 times higher rate of falls $(p=0.003)$ and 1.3 higher rate in women with frailty was reported based 
on the CHS phenotype $(p=0.04)$. Both models had similarly low C index estimates around $0.55(p$ for comparison $=0.97)$.

\section{Discussion}

In this large sample of older women, we demonstrated that self-reported proxy measure for weakness and slowness with a modified level-of-functioning-specific thresholds that was integrated into the WHI frailty phenotype, performed at least as well as the more complex CHS phenotype in predicting important geriatric outcomes. Classification of frailty using the two phenotypes was concordant in $73 \%$ of participants, and the kappa statistic agreement measure was fair to moderate. About $5 \%$ were judged as frail based on either CHS or WHI instruments. A good agreement in terms of prevalence of frailty between the phenotypes confirms the value of the WHI phenotype in screening older women for risk of mortality, falls, and fracture.

The WHI and CHS approaches are different in the objective versus the subjective evaluation of functional capacity. In the CHS phenotype, functional performance is measured by grip strength and timed walking-speed tests; in the WHI phenotype functional capacity is measured by self-report. Because subject-completed measures rely on self-perception of functional performance difficulties, they are likely to indicate, to a certain extent, participants' superior knowledge of their own health over objective measures. If this is the case, the subjective assessment is not necessarily a disadvantage given a well-established association between self-rated health and adverse outcomes (13-15). This conclusion resonated in recent analysis that demonstrated that operationalization of frailty in which selfreport tool substituted for slowness measure yielded a better prediction of mortality within 5 years of follow up (16).

Although the C-statistics show that the predictive ability of the two models is similar, it seems that the WHI phenotype had a higher sensitivity for prefrail group in predicting falls and for frail women in regards to incidents hip fracture. These findings are somehow expected because performance in the activities of daily living (ADL) is reflected in the selfreport measure. ADL performance has been shown to be a strong predictor of falls (17) and inclusion of these measures could be informative in risk assessment as reflected in frailty measure. Additionally, absolute rather than relative thresholds used to define low functioning, could contribute to higher sensitivity of the WHI phenotype

This study has several strengths, including a large sample size, careful adjudication of outcomes, and completeness of a long-term follow-up. We also acknowledge several limitations. First, the measures used to define components of the CHS phenotype in this analysis were similar but not identical to those used in the original definition. In slowness criteria we used a general cutoff point that was indicative of mobility impairment in other studies $(9,10)$ rather than using a sample specific threshold, and exhaustion items were different but conceptually identical to those used in Fried's definition. Second, older women with low levels of functioning and health were less likely than women with better functioning to participate in performance based assessments and complete self reported questionnaires. Loss to follow up may underestimate the proportion of frail women at risk 
for adverse events. However, rather than determining epidemiology of frailty, the aim of this analysis was to provide a head-to-head comparison between commonly used screening tools for frailty in terms of their predictive capacity. Third, self-reported nature of the WHI phenotype and falls may imply that participants who misreport weakness might also misreport falls, leading to over estimation of the strength of association. However, the fact that WHI frailty phenotype was also associated with higher rates of hip fracture lends support to the validity of the association with falls. Finally, although using proxy measures for weakness and slowness might be less informative for biological research, simplicity might be advantageous for clinical purposes.

In summary, the easily collected and scored modified WHI phenotype provides an operational definition of frailty whose predictive ability for falls, hip fractures, and mortality is at least as good as that of the more complex CHS phenotype. Therefore, WHI phenotype, which does not require direct measurements of physical performance, might offer a practical advantage for epidemiological and public health needs.

\section{Supplementary Material}

Refer to Web version on PubMed Central for supplementary material.

\section{Acknowledgments}

The authors thank the Women's Health Initiative (WHI) investigators and staff for their dedication and the study participants for making the program possible. A listing of WHI investigators can be found at: https://cleo.whi.org/ researchers/Documents\%20\%20Write\%20a\%20Paper/WHI\%20Investigator\%20Short\%20List.pdf

Funding: The WHI program is funded by the National Heart, Lung and Blood Institute, National Institute of Health, U.S. Department of Health, and Human Services through Contracts N01WH22110, 24152, 32100-2, 32105-6, 32108-9, 32111-13, 32115, 32118-32119, 32122, 42107-26, 42129-32, and 44221.

Sponsor's Role: The funding agencies had no role in the design and conduct of this study, the analysis or interpretation of the data, or the preparation of the manuscript.

\section{References}

1. Fried LP, Tangen CM, Walston J, et al. Frailty in older adults: Evidence for a phenotype. J Gerontol A Biol Sci Med Sci. 2001; 56A:M146-156. [PubMed: 11253156]

2. Ensrud KE, Ewing SK, Taylor BC, et al. Frailty and risk of falls, fracture, and mortality in older women: The study of osteoporotic fractures. J Gerontol A Biol Sci Med Sci. 2007; 62A:744-751. [PubMed: 17634322]

3. Avila-Funes JA, Helmer C, Amieva H, et al. Frailty among community-dwelling elderly people in France: The three-city study. J Gerontol A Biol Sci Med Sci. 2008; 63A:1089-1096. [PubMed: 18948560]

4. Woods NF, LaCroix AZ, Gray SL, et al. Frailty: Emergence and consequences in women aged 65 and older in the Women's Health Initiative Observational Study. J Am Geriatr Soc. 2005; 53:13211330. [PubMed: 16078957]

5. Johansen KL, Dalrymple LS, Delgado C, et al. Comparison of self-report-based and physical performance-based frailty definitions among patients receiving maintenance hemodialysis. Am J Kid Dis. 2014; 64:600-607. [PubMed: 24793033]

6. Kutner NG, Zhang R. Frailty in dialysis-dependent patients with end-stage renal disease. JAMA Intern Med. 2013; 173:78-79. [PubMed: 23318591] 
7. Anderson GL, Manson J, Wallace R, et al. Implementation of the Women's Health Initiative study design. Ann Epidemiol. 2003; 13:S5-S17. [PubMed: 14575938]

8. Ware JE Jr, Sherbourne CD. The MOS 36-item short-form health survey (SF-36). I Conceptual framework and item selection. Med Care. 1992; 30:473-483. [PubMed: 1593914]

9. Studenski SA, Peters KW, Alley DE, et al. The FNIH sarcopenia project: Rationale, study description, conference recommendations, and final estimates. J Gerontol A Biol Sci Med Sci. 2014; 69:547-558. [PubMed: 24737557]

10. McLean RR, Shardell MD, Alley DE, et al. Criteria for clinically relevant weakness and low lean mass and their longitudinal association with incident mobility impairment and mortality: The foundation for the National Institutes of Health (FNIH) sarcopenia project. J Gerontol A Biol Sci Med Sci. 2014; 69:576-583. [PubMed: 24737560]

11. Harrell FE, Lee KL, Mark DB. Multivariable prognostic models: Issues in developing models, evaluating assumptions and adequacy, and measuring and reducing errors. Stat Med. 1996; 15:361-387. [PubMed: 8668867]

12. Newson RB. Comparing the predictive power of survival models using Harrell's C or Somers' D. Stata J. 2010; 10:339-358.

13. Ashburner JM, Cauley JA, Cawthon P, et al. Self-ratings of health and change in walking speed over 2 years: Results from the caregiver-study of osteoporotic fractures. Am J Epidemiol. 2011; 173:882-889. [PubMed: 21354990]

14. Brenowitz WD, Hubbard RA, Crane PK, et al. Longitudinal associations between self-rated health and performance-based physical function in a population-based cohort of older adults. PLoS One. 2014; 9:e111761. [PubMed: 25365288]

15. Idler EL, Benyamini Y. Self-rated health and mortality: A review of twenty-seven community studies. J Health Soc Behav. 1997; 38:21-37. [PubMed: 9097506]

16. Theou O, Cann L, Blodgett J, et al. Modifications to the frailty phenotype criteria: Systematic review of the current literature and investigation of 262 frailty phenotypes in the Survey of Health, Ageing, and Retirement in Europe. Ageing Res Rev. 2015; 21:78-94. [PubMed: 25846451]

17. Perell KL, Nelson A, Goldman RL, et al. Fall risk assessment measures: An analytic review. J Gerontol A Biol Sci Med Sci. 2001; 56:M761-766. [PubMed: 11723150] 


\section{Table 1}

\section{Characteristics of 3,558 Female Participants Aged 65 Years and Older}

\begin{tabular}{|c|c|}
\hline Characteristics & Value \\
\hline Age, yr $(S D)$ & $70.91(3.68)$ \\
\hline \multicolumn{2}{|l|}{ Frailty status according to CHS phenotype, $n(\%)$} \\
\hline Nonfrail & $1,539(43.3)$ \\
\hline Prefrail & $1,847(51.9)$ \\
\hline Frail & $172(4.8)$ \\
\hline \multicolumn{2}{|c|}{ Frailty status according to WHI phenotype, $n(\%)$} \\
\hline Nonfrail & $1,428(40.1)$ \\
\hline Prefrail & $1,950(54.8)$ \\
\hline Frail & $180(5.1)$ \\
\hline \multicolumn{2}{|l|}{ Individual CHS phenotype components, $n(\%)$} \\
\hline Weakness & $952(26.8)$ \\
\hline Slowness & $379(10.7)$ \\
\hline Poor energy & $258(7.3)$ \\
\hline Low physical activity & $693(19.5)$ \\
\hline Weight loss & $632(17.8)$ \\
\hline \multicolumn{2}{|l|}{ Individual WHI phenotype components, $n(\%)$} \\
\hline Low physical function (2 points $)^{a}$ & $33(1.00)$ \\
\hline Intermediate physical function (1 point) $b$ & $1,454(40.9)$ \\
\hline Good physical function (0 points) ${ }^{c}$ & $2,071(58.2)$ \\
\hline Poor energy & $258(7.3)$ \\
\hline Low physical activity & $693(19.5)$ \\
\hline Weight loss & $632(17.8)$ \\
\hline \multicolumn{2}{|l|}{ Study outcomes } \\
\hline Death, $n(\%)$ & $749(21.1)$ \\
\hline Incident hip fracture, $n(\%)$ & $157(4.4)$ \\
\hline Incident fall, $n(\%)$ & $981(27.6)$ \\
\hline
\end{tabular}

Notes $:$ CHS $=$ Cardiovascular Health Study; WHI = Women's Health Initiative.

${ }^{a}$ RAND-36 Physical Function scores lower than 13

$b_{\text {RAND-36 Physical Function scores between 13-78 }}$

${ }^{c}$ RAND-36 Physical Function scores higher than 78 
Table 2

\section{Concordance between CHS and WHI Phenotypes in Levels of Frailty}

\begin{tabular}{lrrr}
\hline & \multicolumn{3}{c}{ CHS phenotype } \\
\cline { 2 - 4 } WHI phenotype & Nonfrail & Prefrail & Frail \\
\hline Nonfrail & 1,087 & 341 & 0 \\
Prefrail & 452 & 1,418 & 80 \\
Frail & 0 & 88 & 92 \\
\hline
\end{tabular}

Notes: kappa $=0.5(p<0.001)$; CHS $=$ Cardiovascular Health Study; WHI $=$ Women's Health Initiative 


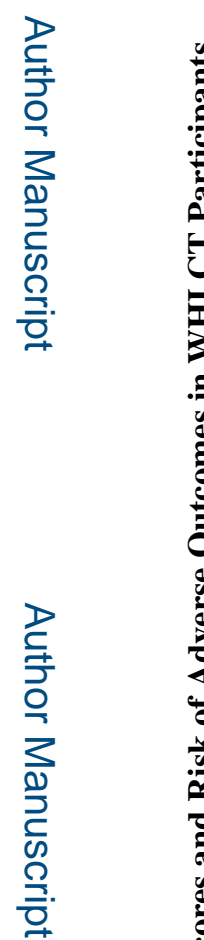

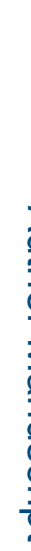

讋

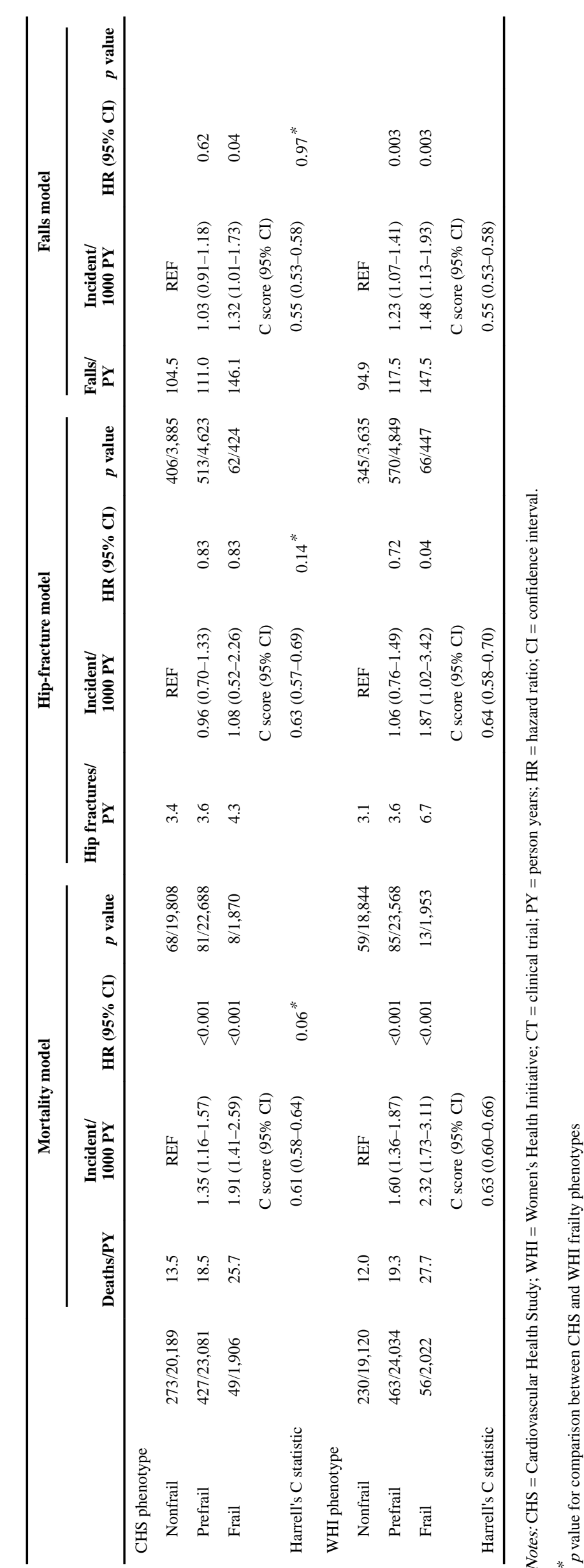

